\title{
Heat Transfer Coefficient Estimation for Turbulent Boundary Layers
}

\author{
S. Wang ${ }^{1}$, Y. Xia ${ }^{1}$, W. Abu Rowin ${ }^{1}$, I. Marusic ${ }^{1}$, R. Sandberg ${ }^{1}$, D. Chung ${ }^{1}$, N. Hutchins ${ }^{1}$, K. Tanimoto ${ }^{2}$ and T. Oda $^{2}$, \\ ${ }^{1}$ Department of Mechanical Engineering \\ University of Melbourne, Victoria 3010, Australia \\ 2 Research Innovation Center \\ Takasago, Mitsubishi Heavy Industries Ltd., Japan
}

\begin{abstract}
Convective heat transfer in rough wall-bounded turbulent flows is prevalent in many engineering applications, such as in gas turbines and heat exchangers. At present, engineers lack the design tools to accurately predict the convective heat transfer in the presence of non-smooth boundaries. Accordingly, a new turbulent boundary layer facility has been commissioned, where the temperature of an interchangeable test surface can be precisely controlled, and conductive heat losses are minimized. Using this facility, we can estimate the heat transfer coefficient (Stanton number, $S t$ ), through measurement of the power supplied to the electrical heaters and also from measurements of the thermal and momentum boundary layers evolving over this surface. These methods have been initially investigated over a shorter smooth prototype heated surface and compared with existing $S t$ prediction models. Preliminary results suggest that we can accurately estimate $S t$ in this facility.
\end{abstract}

\section{Keywords}

Heat transfer; Boundary layers; Heat transfer coefficient

\section{Introduction}

When a moving fluid comes into contact with a stationary solid surface and where a temperature difference exists between the fluid and solid, convective heat transfer occurs. The heat transfer coefficient is defined by the Stanton number $(S t)$, which can be considered as the heat transfer analogue to the skin-friction coefficient $\left(C_{f}\right)$. Kays \& Crawford [3] and White \& Corfield [11] derived the $S t$ equation from an analogue to the Clauser chart based on the inner scaled mean temperature profile. Kays \& Crawford [3] also derived a relationship between St and enthalpy thickness based on the energy integral equation. Ligrani \& Moffat and Mukerji et al. [5, 7] estimated St from an energy balance performed on each segment of an electrically heated plate. Ligrani \& Moffat [5] used a power meter to measure the total electrical power, estimating convective heat flux as the total electrical power minus the heat losses due to conductive and radiative heat transfer. Mukerji et al. [7] used calorimeters to measure the wall heat flux directly.

The above-cited studies developed expressions to estimate $S t$ for turbulent boundary layers over heated smooth surfaces. However, in many engineering applications, the surfaces are non-smooth, which, for some of the above methods, adds complexity for estimating St. To study the influence of surface roughness on heat transfer, a turbulent boundary layer flow facility is under development with a controllable constant temperature test surface (that can be either smooth or rough). Here we describe the design of the facility and test several techniques to compute the local $S t$ over a heated smooth surface to validate the design and proposed instrumentation.

\section{Estimating heat transfer coefficient}

We consider three methods to estimate the local Stanton number
$(S t)$. The first method computes $S t$ from the following expression using a direct measurement of the thermal and momentum boundary layers

$$
S t=\frac{\Theta_{\tau}}{\Theta_{w}-\Theta_{\infty}} \sqrt{\frac{C_{f}}{2}},
$$

where, $\Theta_{w}$ is the wall temperature, $\Theta_{\infty}$ is the free stream temperature and $\Theta_{\tau}$ is the friction temperature estimated from the Clauser fit to the thermal boundary layer (discussed in the results section below). The skin friction coefficient $C_{f}$ in equation (1) is evaluated from

$$
C_{f}=2\left(\frac{U_{\tau}}{U_{\infty}}\right)^{2}
$$

where $U_{\tau}$ is the friction velocity, estimated from a Clauser fit to the momentum boundary layer, and $U_{\infty}$ is the freestream velocity. There are concerns over the accuracy of this approach, especially for rough walls, where the Clauser fit involves additional fitting parameters (the Hama roughness function $\Delta U^{+}$, its thermal analogue $\Delta \Theta^{+}$and also a virtual wall-normal origin) which increases the error. Note from equation (1), that this estimate for $S t$ will have compounded errors due to the application of two modified Clauser fits.For the present low Reynolds number conditions, we estimate that the error in $S t$ calculated via Clauser-type fits to equation (1) will be $\pm 9 \%$.

The second method is through measurement of the convective heat flux, $q_{c}^{\prime \prime}$. The relationship between the Stanton number and forced convective heat flux is

$$
S t=\frac{q_{c}^{\prime \prime}}{\rho C_{p} U_{\infty}\left(\Theta_{w}-\Theta_{\infty}\right)} .
$$

Here, $\rho$ is the working fluid density and $C_{p}$ is the fluid specific heat at constant pressure.

The rate of change of the enthalpy thickness along the streamwise direction, $x$, can also be used to compute $S t$. The derivation here (as described in [3,11]) is similar to von Kármán's mean momentum balance which for zero pressure gradient boundary layers yields the result $C_{f} / 2=\mathrm{d} \theta / \mathrm{d} x$ ( $\theta$ is the momentum thickness and $x$ is the streamwise direction). Through integrating the applicable form of the momentum and energy equations for an isothermal surface [3], the Stanton number is given by $S t=\mathrm{d} \delta_{h} / \mathrm{d} x$, where $\delta_{h}$ is the enthalpy thickness defined as [11]

$$
\delta_{h}=\int_{0}^{\infty} \frac{U}{U_{\infty}}\left(\frac{\Theta-\Theta_{\infty}}{\Theta_{w}-\Theta_{\infty}}\right) \mathrm{d} z .
$$

Here, $U$ and $\Theta$ are the mean streamwise velocity and temperature respectively (which for given operational conditions will be a function of $z, x$ ) and $z$ is the wall-normal distance. This method is not assessed for the experiments reported here over a heated plate of limited streamwise fetch as it requires temperature measurement at several streamwise locations. 


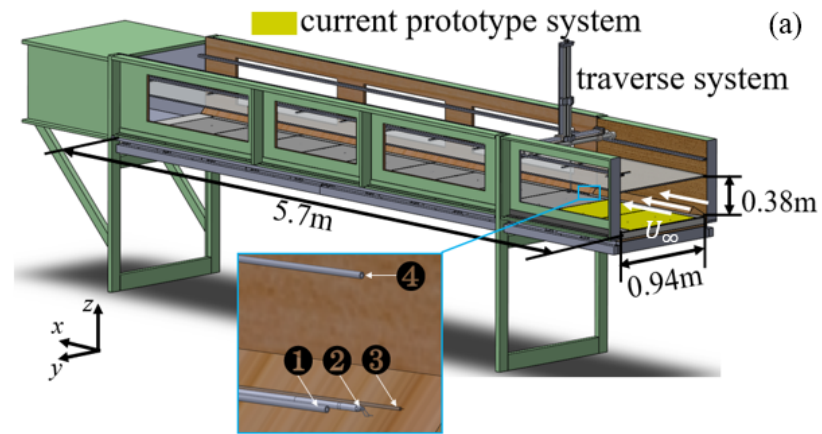

(a)

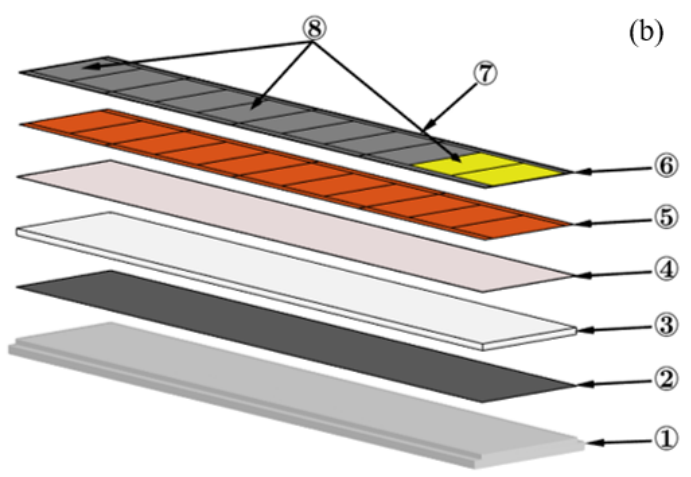

Figure 1. (a) Schematic showing the tunnel, the heated surfaces and the three sensors installed on the traverse system, (1 static pressure tube, (2) hot-wire, 3 thermocouple, 4 total head tube; (b) exploded view of the wind tunnel floor structure, (1) wooden floor, (2) steel sheet, (3) polyisocyanurate foam insulation plate, (4) calcium-magnesium silicate thermal insulation sheet, (5) pad heater, (6) aluminum plate, (7) side rail, (8) main plate,

\section{Test section design}

Experiments are conducted in a boundary-layer wind tunnel (illustrated in figure 1a) of working section $5.7 \times 0.94 \times 0.38 \mathrm{~m}^{3}$ in $x \times y \times z$ directions. For more details about the facility, the reader is referred to [9]. Measurements are made in the turbulent boundary layer developed over the tunnel floor. To provide a temperature difference between the solid boundary and the fluid, a new floor has been designed consisting of eleven $720 \times 500 \mathrm{~mm}^{2}(x \times y)$ heated $6.35 \mathrm{~mm}$ thick anodized smooth ALCA5 aluminum cast plates (see figure 1). Each plate is heated by a custom made heater pad (Holroyd Components Ltd) with a power of $1.22 \mathrm{~kW}$ each $\left(3.4 \mathrm{~kW} / \mathrm{m}^{2}\right)$. The floor temperature is monitored through thermocouples with $0.05^{\circ} \mathrm{C}$ uncertainty embedded in the aluminum plates. Power to each heater is controlled individually with solid-state relays and a proportional-integral-derivative (PID) controller system to maintain the floor at a constant temperature. The power consumption for each heater is recorded with a Powertech MS-6108 power meter with $41 \mathrm{~W}$ measurement uncertainty. Ideally, the majority of this measured power is transferred to the fluid via convective heat transfer, for which the design needs to minimise conductive heat loss. The heat loss through the floor of the tunnel is minimized with a $53 \mathrm{~mm}$ thick MetecnoPanel rigid polyisocyanurate foam insulation plate $(0.02 \mathrm{~W} /(\mathrm{m} \cdot \mathrm{K}))$ and an additional $3 \mathrm{~mm}$ thick calcium-magnesium silicate thermal insulation sheet $(0.232 \mathrm{~W} /(\mathrm{m} \cdot \mathrm{K}))$. Additional thermocouples on the bottom outer surface of the wind-tunnel are used to calculate the conduction heat loss from the bottom of the heated plates. As suggested by Hosni et al. [2], the conductive heat loss to the tunnel side walls is also reduced with a series of heated side-rails that are maintained at the same temperature as the test plates. An exploded view of the heated test-surface is provided in figure 1(b).

\section{Initial experiments over prototype surface}

All measurements presented here are conducted in zeropressure-gradient turbulent boundary layers formed over a (heated and unheated) smooth surface at a streamwise distance $x=800 \mathrm{~mm}$ from the tripped inlet of the working section. It is emphasized that this is a test of the prototype surface, consisting of just two test plates covering the first meter downstream of the tripped inlet to the working section. For this test there were no heated side-rails, and the under-insulation consisted of $3 \mathrm{~mm}$ thick calcium-magnesium silicate type insulation material, hence the conductive heated losses were much larger in this case. This prototype tested surface is indicated by the yellow shaded regions in figure 1. Three sensors, installed on a traverse system, are utilized to measure velocity and temperature along the wall-normal direction as displayed in figure 1(a). The encoder of the traverse system has uncertainty of $5 \mu \mathrm{m}$. A single hot-wire sensor is used to obtain the instantaneous velocity. Since the thermal boundary layer affects the hot-wire measurement, a Pitot-static tube is also used to provide a reference mean velocity measurement as it is relatively insensitive to the temperature changes. The free stream velocity uncertainty is $0.025 \mathrm{~m} / \mathrm{s}$ due to the pressure transducer. An E-type thermocouple is also included on the traverse system for temperature measurement. All measurements are performed at a constant free-stream velocity, $U_{\infty}=20 \mathrm{~ms}^{-1}$ corresponding to friction Reynolds number, $R e_{\tau}=U_{\tau} \delta / v \approx 1050$, where $\delta$ is the thickness of the boundary layer and $v$ is the kinematic viscosity. A summary of the measurement parameters of the unheated and heated cases is included in table 1 . The uncertainty of the measurement is summarized in table 2 .

\begin{tabular}{lcccccc}
\hline & $U_{\infty}$ & $\delta$ & $v \times 10^{5}$ & $U_{\tau}$ & $R e_{\tau}$ & $\Theta_{w}-\Theta_{\infty}$ \\
\hline Unit & $(\mathrm{m} / \mathrm{s})$ & $(\mathrm{mm})$ & $\left(\mathrm{m}^{2} / \mathrm{s}\right)$ & $(\mathrm{m} / \mathrm{s})$ & & $\left({ }^{\circ} \mathrm{C}\right)$ \\
Unheated & 20 & 21.7 & 1.60 & 0.794 & 1075 & 0 \\
Heated & 20 & 21.8 & 1.58 & 0.762 & 1053 & 15 \\
\hline
\end{tabular}

Table 1. Parameters of unheated and heated smooth surfaces.

\begin{tabular}{lccccccc}
\hline Parameter & Power & $U_{\infty}$ & $\Theta_{w}$ & $\Theta_{\infty}$ & $z$ & $U_{\tau}$ & $\theta_{\tau}$ \\
\hline Unit & $(\mathrm{W})$ & $(\mathrm{m} / \mathrm{s})$ & $\left({ }^{\circ} \mathrm{C}\right)$ & $\left({ }^{\circ} \mathrm{C}\right)$ & $(\mu \mathrm{m})$ & $(\mathrm{m} / \mathrm{s})$ & $\left({ }^{\circ} \mathrm{C}\right)$ \\
$(\%)$ & 13.2 & 0.1 & 0.1 & 0.8 & 0.1 & 6.1 & 7.4 \\
Uncertainty & 41 & 0.025 & 0.05 & 0.2 & 5 & 0.048 & 0.059 \\
\hline
\end{tabular}

Table 2. Uncertainty of the measurement.

\section{Results}

Initial experiments with the PID controller active, used a thermal imaging system and multiple distributed embedded and calibrated thermocouples to ensure that the prototype heated plate design and controller could maintain a constant wall temperature over the range of flow conditions, with no apparent discontinuity at the interface between plates. Figure 2(a) shows the mean streamwise velocity profiles, $U^{+}=U / U_{\tau}$, of the unheated surface from both the hot-wire and Pitot-tube, with good overall agreement. Here $U_{\tau}$ is estimated from a Clauser chart fit with the logarithmic profile of $U^{+}=\kappa^{-1} \ln z^{+}+4.17$, where 
$\kappa=0.384$ is the von Kármán constant. The velocity profiles also overlap with the reference profiles of the numerical simulation of Schlatter \& Orlu [10] at a comparable $R e_{\tau}=974$ The hot-wire and Pitot-tube measured velocity profiles are com-
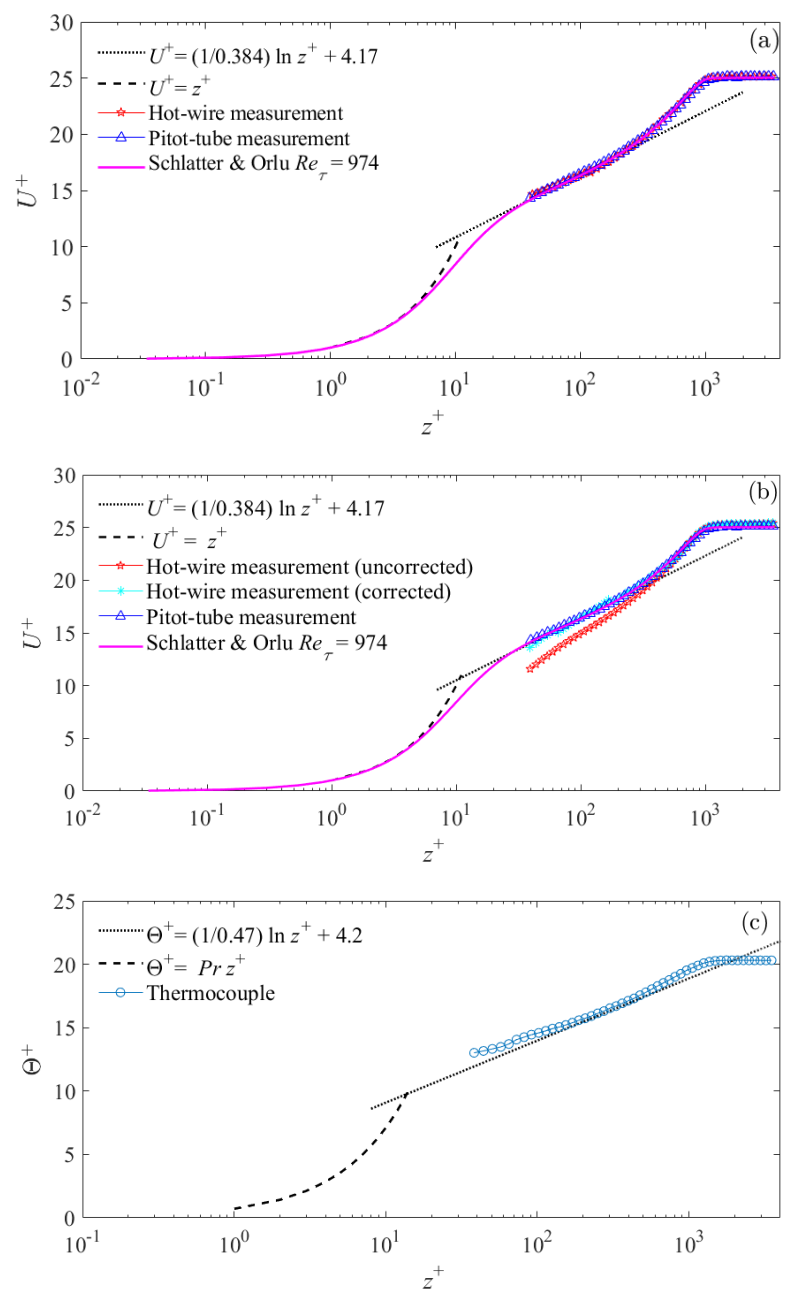

Figure 2. (a) Mean velocity of unheated surface measured by hot-wire and Pitot-tube. A profile from the DNS study of Schlatter \& Orlu [10], law-of-the-wall $U^{+}=z^{+}$, and the log-law are included for comparison; (b) mean velocity of heated surface measured by hot-wire and Pitottube; (c) mean temperature profile of heated surface.

pared for the heated plate in figure 2(b). The Pitot-tube measured profile retains good agreement with the numerical profile of Schlatter \& Orlu [10] in the buffer and log-law regions indicating that the heating has no effect on the momentum boundary layer and that this is pure forced convection, with no buoyancy effects (as confirmed from the calculation of the stability parameter below). As expected, the mean velocity measured by the hot-wire for the heated case is lower than the unheated case at $z^{+}<500$, while it is comparable to the numerical profile further away from the wall. The discrepancy between the Pitottube and the hot-wire measurements is related to the effect of elevated near wall temperatures on the hot-wire operation (near the wall, the effective overheat ratio of the hot wire is reduced which alters the calibration curve). To correct the hot-wire for temperature drift near the heated surface in figure 2(b), we need to measure the mean temperature in the boundary layer. The mean inner-scale temperature profile, $\Theta^{+}=\left(\Theta_{w}-\Theta\right) / \Theta_{\tau}$, for the smooth heated wall is shown in figure $2(\mathrm{c})$. The friction temperature $\Theta_{\tau}$ is estimated from fitting the inner-scaled mean temperature profile to the logarithmic temperature profile defined as [11]

$$
\Theta^{+}=\frac{1}{\kappa_{h}} \ln \left(z^{+}\right)+A_{r}(\operatorname{Pr}),
$$

here $\kappa_{h}=\kappa / P r_{t}=0.47$ is the slope coefficient (where $P r_{t}$ is the turbulent Prandtl number assumed to be 0.85 [11]) and $A_{r}=4.2$ for air $(\operatorname{Pr}=0.71[11])$. The error in this fit is estimated at $\pm 7 \%$ based on uncertainties in $\kappa_{h}, A_{r}$ and the log linear fit.

The hot-wire voltage can be corrected by following the procedure suggested by Bruun[1]

$$
E_{\text {corr }}^{2}=E^{2}\left[1-0.5 \frac{\Theta-\Theta_{\infty}}{\Theta_{\text {wire }}-\Theta_{\infty}}\right]
$$

where, $E_{c o r r}$ is the correct hot-wire voltage, $E$ is the original hot-wire voltage and $\Theta_{\text {wire }}$ is the hot-wire temperature estimated by the sensor temperature coefficient of resistance and the overheat ratio [1]. The corrected HWA measured mean velocity profile for the heated case is shown by the light blue symbols in figure 2(b), with much improved overall agreement with the Pitot-static and DNS results. The discrepancy of $U^{+}$at $z^{+}<40$ could be related to the limited resolution of the thermocouple measurement near the wall, and will be improved in the future through use of cold-wires, which offer much finer wallnormal resolution. Nevertheless, figure 2 gives confidence that the mean velocity profiles measured by HWA in thermal boundary layers can be corrected from the mean temperature profile, and also that the momentum boundary layer in this case (where the stability parameter is close to zero), can be measured over the unheated plate with little loss of accuracy.

The stability parameter can be calculated from the preliminary measurements to confirm that buoyancy effects are small. We use a Monin-Obukhov stability parameter $\left|\delta_{T} / L\right|$, where $\delta_{T}$ is the thermal boundary layer thickness determined from figure 2(c), and $L$ is the Obukhov length, which is calculated by

$$
L=\frac{-U_{\tau}^{3} \Theta_{w}}{\kappa g q_{c}^{\prime \prime}}
$$

where, $g$ is the gravitational acceleration. For the expected range of experimental conditions $(P r=0.71)$, the largest stability parameter occurs at the edge of the thermal layer where $\left|\delta_{T} / L\right|=0.0018$ which is much smaller that the generally accepted limits that indicate a negligible buoyancy effect. Krug et al. [4] have recently suggested that the aspect ratio of the hierarchical turbulent structure can be extremely sensitive to the stability parameter. A full heating power, the proposed test facility will be able to produce weakly unstable boundary layers with a stability parameter as high as 0.02 , which Krug et al. [4] suggest will alter the aspect ratio of turbulent structures from $14: 1$ (streamwise:wall-normal) to as low as $8.3: 1$, suggesting that this facility can be used to systematically investigate this effect.

Evaluating the Stanton number using equation (1) requires an estimate for the skin friction coefficient $\left(C_{f}\right)$ which is calculated using equation (2), where $U_{\tau}$ is given by a Clauser fit to the mean velocity profile. The evolution of $C_{f}$ evaluated in this manner is found to be in good agreement with that shown by Nagib \& Chauhan [8]. The resulting estimate for St is shown by the black symbol on figure 3 . We can also estimate $S t$ from the convective heat flux of equation (3). Based on the uncertainty shown in table 2, the error in the Stanton number assessed by the error propagation method is estimated as $\pm 16 \%$ for the measured power method and $\pm 9 \%$ for the Clauser method, as shown by the red and black error bars respectively in figure 3 . As explained by Moffat et al. [6], the convective heat flux, $q_{c}^{\prime \prime}$, is 
obtained from the difference between the total power consumption measured by the power meter and the predicted heat loss due to conduction from the tunnel bottom and side walls, as well as the radiative heat loss. To estimate the heat loss from the tunnel floor, the exterior temperature of the tunnel floor was first measured, from which the total conductive heat loss through the tunnel floor was estimated at $60 \mathrm{~W} / \mathrm{m}^{2}$. The radiative heat losses $q_{r l}^{\prime \prime}$, modeled as a grey body, are estimated using $q_{r l}^{\prime \prime}=\varepsilon \sigma\left(\Theta_{w}^{4}-\Theta_{\infty}^{4}\right)$ where, $\varepsilon$ is anodised aluminum emissivity 0.77 and $\sigma$ is the Stefan-Boltzmann constant $5.67 \times 10^{-8}$ $\mathrm{W} / \mathrm{m}^{2} \mathrm{~K}^{4}$. The radiative heat loss is estimated as $0.11 \mathrm{~W} / \mathrm{m}^{2}$, which amounts to approximately $0.014 \%$ of the convective heat loss for the current experiments. The non-convective heat loss is dominated by the conductive heat loss from wind tunnel bottom, which amounts to approximately $7.5 \%$ of the convective heat loss for the current experiments, but will be substantially reduced to approximately $0.2 \%$ with the inclusion of the $53 \mathrm{~mm}$ polyisocyanurate foam insulation plate in the full-scale system. Without the heated side rails, the conductive side heat loss is estimated to be $20 \%$ of the floor heat loss. The computed $S t$ from the two methods (Clauser fit and measured power) are plotted against the streamwise Reynolds number, $R e_{x}=x U_{\infty} / v$ in figure 3. Models of $S t$ from [3, 5, 7] are also included in Figure 3 for comparison. Ligrani \& Moffat and Mukerji et al. $[5,7]$ corrected the predicted $S t$ evolution by considering the unheated starting length $\xi$. For these experiments the unheated starting length is estimated from the evolution of the boundary layer thickness $\delta$ as a function of $x$, with $\xi$ assumed to be the streamwise location where the projected fit to $\delta(x)$ reaches zero (which is upstream of the working section). Besides the unheated length, Mukerji et al. [7] introduced a new term $\phi$ related to $\xi$, streamwise location $x$, and the unheated length Reynolds number $R e_{\xi}=\xi U_{\infty} / v$ to correct St. The St calculated from equation (1) falls on the reference models of [5, 7]. $S t$ calculated from $q_{c}^{\prime \prime}$, however, is slightly larger than the reference models which presumably is related to the accuracy of the power meter or the estimation of the heat loss. The current power meter will be upgraded for better accuracy for future work, and conductive heat losses will be minimised once measurements are made over the complete surface as illustrated in figure 1(b). We will also have opportunities to perform the energy balance as described in equation (4) to provide a further estimate of $S t$.

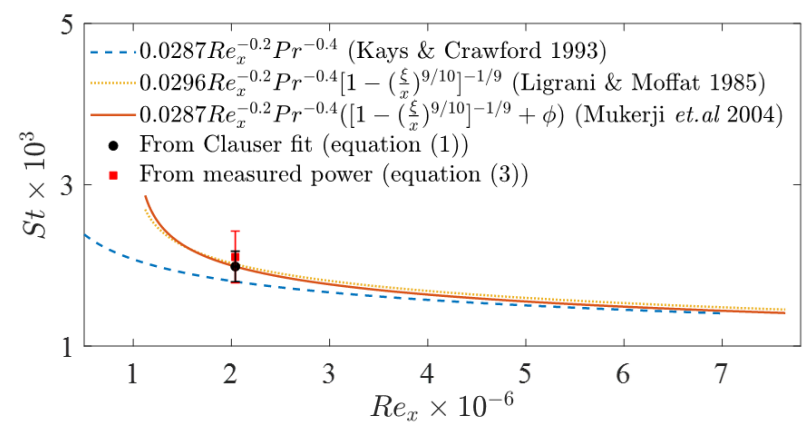

Figure 3. Stanton number of heated smooth surface.

\section{Conclusion}

A modular system for a heated test surface has been described, which permits investigation of forced convective heat transfer in smooth- and rough-wall bounded turbulent flows. The proposed design provides accurate isothermal wall conditions, and minimises losses due to conduction. Preliminary validating measurements are performed over a prototype version of the design, with limited streamwise fetch and without the heated side rails and underfloor insulation. For these experiments we used a traversable sensor package consisting a Pitot tube, hotwire and a thermocouple to measure mean velocity and temperature profiles. Initial results confirm that for the operating conditions tested (excess wall tempertature $\Theta_{w}-\Theta_{\infty}=15^{\circ} \mathrm{C}$, $U_{\infty}=20 \mathrm{~ms}^{-1}$ ), this is an almost purely convective regime, with no discernible buoyancy effects (the heated fluid acts as a passive scalar). Results also indicate that the hot-wire measurement drift due to the thermal boundary layer can be corrected for the mean velocity profile using the mean temperature profile. The facility was validated by computing the heat transfer coefficient over a heated smooth surface with two techniques and compared with existing predictive models for the evolution of St. The results were consistent with the predictive models suggesting that, with the further improvements offered by the full-scale system, this facility can be used for investigations of forced convective heat transfer over rough and smooth surfaces.

\section{Acknowledgements}

The authors acknowledge support from the Australian Research Council via grants LP180100712 and DP200100969.

\section{References}

[1] Bruun, H. H., Hot-wire anemometry: principles and signal analysis, IOP Publishing, 1996.

[2] Hosni, M., Coleman, H. W. and Taylor, R. P., Measurements and calculations of rough-wall heat transfer in the turbulent boundary layer, Int. J. Eng. Fluid Mech., 34, 1991, 1067-1082.

[3] Kays, W. M. and Crawford, M. E., Convective Heat and Mass Transfer, McGraw-Hill, 1993.

[4] Krug, D., Baars, W. J., Hutchins, N. and Marusic, I., Vertical coherence of turbulence in the atmospheric surface layer: connecting the hypotheses of townsend and davenport, Boundary-Layer Meteorol, 172, 2019, 199-214.

[5] Ligrani, P. and Moffat, R., Thermal boundary layers on a rough surface downstream of steps in wall temperature, Boundary-Layer Meteorol, 31, 1985, 127-147.

[6] Moffat, R., Healzer, J. and Kays, W., Experimental heat transfer behavior of a turbulent boundary layer on a rough surface with blowing, J. Heat Transf., 100, 1978, 134142.

[7] Mukerji, D., Eaton, J. K. and Moffat, R. J., Convective heat transfer near one-dimensional and two-dimensional wall temperature steps, J. Heat Transf., 126, 2004, 202210.

[8] Nagib, H. M. and Chauhan, K. A., Variations of von kármán coefficient in canonical flows, Phys. Fluids, 20, 2008, 101518.

[9] Nugroho, B., Hutchins, N. and Monty, J. P., Large-scale spanwise periodicity in a turbulent boundary layer induced by highly ordered and directional surface roughness, Int. J. Heat Fluid Flow, 41, 2013, 90-102.

[10] Schlatter, P. and Örlü, R., Assessment of direct numerical simulation data of turbulent boundary layers, Journal of Fluid Mechanics, 659, 2010, 116.

[11] White, F. M. and Corfield, I., Viscous Fluid Flow, McGraw-Hill New York, 2006, 3 edition. 


\section{University Library}

\section{- M M N E R VA A gateway to Melbourne's research publications}

Minerva Access is the Institutional Repository of The University of Melbourne

\section{Author/s:}

Wang, S;Xia, Y;Abu Rowin, W;Marusic, I;Sandberg, R;Chung, D;Hutchins, N;Tanimoto, $\mathrm{K} ; \mathrm{Oda}, \mathrm{T}$

Title:

Heat Transfer Coefficient Estimation for Turbulent Boundary Layers

Date:

2020-12-11

\section{Citation:}

Wang, S., Xia, Y., Abu Rowin, W., Marusic, I., Sandberg, R., Chung, D., Hutchins, N., Tanimoto, K. \& Oda, T. (2020). Heat Transfer Coefficient Estimation for Turbulent Boundary Layers. Proceedings of the 22nd Australasian Fluid Mechanics Conference AFMC2020, The University of Queensland. https://doi.org/10.14264/3969498.

Persistent Link:

http://hdl.handle.net/11343/288707

License:

CC BY-NC 